\title{
Gedanken Experiment for B Field Contributions to Initial Conditions for Relic Graviton Production Based upon an Initial Inflaton Value
}

\author{
Andrew Walcott Beckwith \\ Physics Department, Chongqing University, Chongqing, China \\ Email: abeckwith@uh.edu,rwill9955b@gmail.com
}

How to cite this paper: Beckwith, A.W. (2017) Gedanken Experiment for B Field Contributions to Initial Conditions for Relic Graviton Production Based upon an Initial Inflaton Value. Journal of High Energy Physics, Gravitation and Cosmology, 3, 651-656.

https://doi.org/10.4236/jhepgc.2017.34049

Received: August 17, 2017

Accepted: October 16, 2017

Published: October 19, 2017

Copyright $\odot 2017$ by author and

Scientific Research Publishing Inc.

This work is licensed under the Creative

Commons Attribution International

License (CC BY 4.0).

http://creativecommons.org/licenses/by/4.0/

\begin{abstract}
This paper is to address using what a fluctuation of a metric tensor leads to, in Pre Planckian physics, namely $\delta t \Delta E \geq \frac{\hbar}{\delta g_{t t}} \neq \frac{\hbar}{2}$. If so then, we pick the conditions for an equality, with a small $\delta g_{t t}$, to come up with initial temperature, particle count and entropy affected by initial degrees of freedom in early Universe cosmology. This leads to an initial graviton production due to a minimum magnetic field, as established in our analysis. Which we relate to the inflaton as it initially would be configured and evaluated.
\end{abstract}

\section{Keywords}

Emergent Time, Heavy Gravity, Metric Tensor Perturbations, HUP

\section{Introduction}

This article starts with updating what was done in [1], which is symbolized by, if the scale factor is very small, metric variance [2] [3]

$$
\begin{aligned}
& \left\langle\left(\delta g_{u v}\right)^{2}\left(\hat{T}_{u v}\right)^{2}\right\rangle \geq \frac{\hbar^{2}}{V_{\text {Volume }}^{2}} \\
& \underset{u v \rightarrow t t}{\longrightarrow}\left\langle\left(\delta g_{t t}\right)^{2}\left(\hat{T}_{t t}\right)^{2}\right\rangle \geq \frac{\hbar^{2}}{V_{\text {Volume }}^{2}} \\
& \& \delta g_{r r} \sim \delta g_{\theta \theta} \sim \delta g_{\phi \phi} \sim 0^{+}
\end{aligned}
$$

In [4] this lead to 


$$
\begin{aligned}
& \delta t \Delta E \geq \frac{\hbar}{\delta g_{t t}} \neq \frac{\hbar}{2} \\
& \text { Unless } \delta g_{t t} \sim O(1)
\end{aligned}
$$

We assume $\delta g_{t t}$ is a small perturbation and look at $\delta t \Delta E=\frac{\hbar}{\delta g_{t t}}$ with

$$
\Delta t_{\text {time }}(\text { initial })=\hbar /\left(\delta g_{t t} E_{\text {initial }}\right)=\frac{2 \hbar}{\delta g_{t t} \cdot g_{* s}(\text { initial }) \cdot T_{\text {initial }}}
$$

This would put a requirement upon a very large initial temperature $T_{\text {initial }}$ and so then, if

$$
\begin{aligned}
& S(\text { initial }) \sim n(\text { particle-count }) \approx g_{* s}(\text { initial }) \cdot V_{\text {volume }} \cdot\left(\frac{2 \pi^{2}}{45}\right) \cdot\left(T_{\text {initial }}\right)^{3} \quad[5] \\
& S(\text { initial }) \sim n(\text { particle-count }) \approx \frac{V_{\text {volume }}}{g_{* s}^{2}(\text { initial })} \cdot\left(\frac{2 \pi^{2}}{45}\right) \cdot\left(\frac{\hbar}{\Delta t_{\text {initial }} \cdot \delta g_{t t}}\right)^{3}
\end{aligned}
$$

And if we can write as given in [2] [3]

$$
V_{\text {volume(initial) }} \sim V^{(4)}=\delta t \cdot \Delta A_{\text {surface-area }} \cdot\left(r \leq l_{\text {Planck }}\right)
$$

The volume in the Pre Planckian regime would be extremely small, i.e. if we are using the convention that Equation (4) holds, then it argues for a very large $g_{s}^{*}$ beyond the value of 102, as given in [5]. In any case, our boundary between the Pre Planckian regime and Planckian, as far as the use of Equation (4) yields a preliminary value of, for a distance less than or equal to Planck Length, of non-zero value, with

$$
10^{20} \leq\left. S(\text { initial }) \sim n(\text { particle-count })\right|_{r \leq l_{P}} \leq 10^{37}
$$

This is also assuming a $\delta t_{\text {initial }} \approx \Delta t_{\text {initial }} \propto$ Plank-time, i.e. at or smaller than the usual Planck time interval.

\section{Counter Pose Hypothesis, by String Theory, for Equation}

The author is aware of the String theory minimum length and minimum time which is different from the usual Planck lengths, but are avoiding these, mainly due to a change in the assumed entropy formulae to read as the square root of the above results, namely [6] [7] [8]

$$
10^{10} \leq\left.\left. S(\text { initial })\right|_{\text {String-Theory }} \sim \sqrt{n(\text { particle-count })}\right|_{r \leq l_{P}} \leq 10^{16}
$$

The above is still non-zero, but it cannot be exactly posited as in the Pre Planckian regime of Space-time, since the minimum length may be larger than Planck Length, i.e. as of the sort given in [8].

\section{Questions as to Refining Both Equation (6) and Equation (7) for More Precise Entropy Bounds}

If from Giovannini [9] we can write 


$$
\delta g_{t t} \sim a^{2}(t) \cdot \phi \ll 1
$$

Refining the inputs from Equation (8) means more study as to the possibility of a non-zero minimum scale factor [10], as well as the nature of $\phi$ as specified by Giovannini [9]. We hope that this can be done as to give quantifiable estimates and may link the non-zero initial entropy to either Loop quantum gravity "quantum bounce" considerations [11] and/or other models which may presage modification of the sort of initial singularities of the sort given in [12]. Furthermore if the non-zero scale factor is correct, it may give us opportunities as to fine tune the parameters given in [10] below:

$$
\begin{aligned}
& \alpha_{0}=\sqrt{\frac{4 \pi G}{3 \mu_{0} c}} B_{0} \\
& \hat{\lambda} \text { (defined) }=\Lambda c^{2} / 3 \\
& a_{\min }=a_{0} \cdot\left[\frac{\alpha_{0}}{2 \hat{\lambda}(\text { defined })}\left(\sqrt{\alpha_{0}^{2}+32 \hat{\lambda}(\text { defined }) \cdot \mu_{0} \omega \cdot B_{0}^{2}}-\alpha_{0}\right)\right]^{1 / 4}
\end{aligned}
$$

where the following is possibly linkable to minimum frequencies linked to $\mathrm{E}$ and $M$ fields [10], and possibly relic Gravitons are generated if

$$
B>\frac{1}{2 \cdot \sqrt{10 \mu_{0} \cdot \omega}}
$$

This can be contrasted with looking at what happens if [13]

$$
\begin{aligned}
& a \approx a_{\min } t^{\gamma} \\
& \Leftrightarrow \phi \approx \sqrt{\frac{\gamma}{4 \pi G}} \cdot \ln \left\{\sqrt{\frac{8 \pi G V_{0}}{\gamma \cdot(3 \gamma-1)}} \cdot t\right\} \\
& \Leftrightarrow V \approx V_{0} \cdot \exp \left\{-\sqrt{\frac{16 \pi G}{\gamma}} \cdot \phi(t)\right\}
\end{aligned}
$$

So as talked about with [14] setting a minimum energy density given by

$$
\rho_{\Lambda} \approx \frac{G\left(E / c^{2}\right)^{2}}{l} l^{-3}=\frac{G E^{6}}{c^{8} \hbar^{4}}
$$

And with the following substitution of

$$
E \underset{\text { Pre-Planckian } \rightarrow \text { Planckian }}{\longrightarrow} \Delta E \sim \frac{\hbar}{\Delta t \cdot\left(\delta g_{t t} \approx a_{\text {min }}^{2} \phi_{\text {initial }}\right)}
$$

Then to first order we would be looking at Equation (12) re written as leading to

$$
\rho_{\Lambda} \sim \frac{G}{c^{8} \hbar^{4}} \cdot\left(\frac{\hbar}{\Delta t \cdot\left(\delta g_{t t} \approx a_{\text {min }}^{2} \phi_{\text {initial }}\right)}\right)^{6}
$$

And if Equation (15) holds,

$$
\Lambda_{\text {initial }} \cdot H_{\text {initial }}^{-2} \approx O(1)
$$

we would have by [15] 


$$
\begin{aligned}
& \Lambda_{\text {initial }} \approx H_{\text {initial }}^{2} \sim \gamma^{2} / t^{2} \\
& \Lambda_{\text {initial }} \cdot L_{p}^{2} \approx 10^{-123}
\end{aligned}
$$

So

$$
10^{-123} \sim \gamma^{2} L_{P}^{2} \cdot\left(\Delta E \cdot \delta g_{t t}\right)^{2} / \hbar^{2}
$$

Equation (17) would be key to the entire business, i.e. using this, we would have if

$$
\Delta E \sim \hbar \omega_{\text {graviton }}
$$

Then

$$
10^{-123} \sim \gamma^{2} L_{P}^{2} \cdot\left(\hbar \omega_{\text {graviton }} \cdot \delta g_{t t}\right)^{2} / \hbar^{2} \sim \gamma^{2} L_{P}^{2} \cdot\left(\omega_{\text {graviton }} \cdot \delta g_{t t}\right)^{2}
$$

Then if we go to Equation (10) we have a threshold magnetic field for the production of gravitons which looks like if we apply the minimum scale factor condition [16], that

$$
\begin{aligned}
& B_{\min } \geq \frac{1}{2 \cdot \sqrt{10 \mu_{0} \cdot \omega}} \approx \frac{\sqrt{\gamma L_{P} \delta g_{t t}} 10^{123 / 4}}{2 \cdot \sqrt{10 \mu_{0}}} \approx \frac{\sqrt{\gamma L_{P} \phi_{\text {initial }}} a_{\text {min }} 10^{123 / 4}}{2 \cdot \sqrt{10 \mu_{0}}} \\
& \& a_{\min } \sim\left(10^{-123 / 4}\right) \Rightarrow B_{\min } \geq \frac{\sqrt{\gamma L_{P} \phi_{\text {initial }}}}{2 \cdot \sqrt{10 \mu_{0}}}
\end{aligned}
$$

i.e. we get graviton production if the last line of Equation (20) is satisfied, which means that the initial value of the inflaton, in this case is crucially important.

With that initial inflaton value determined in part by Equation (11).

\section{Conclusions: The Inflaton Minimum Helps Determine a Lower Bound for a Cosmological Initial Production of Gravitons}

The last line of Equation (20) helps establish a minimum magnetic field for the production of relic gravitons, with a magnetic field established through Equation (10) and subsequently modified by Equation (20).

This adds substance to what was brought up by Beckwith in [16] namely that we have a minimum scale factor of

$$
\begin{aligned}
& a_{\min } \sim\left(10^{-123 / 4}\right) \sim\left(\Delta E / E_{P}\right)^{3 / 2} \\
& \& \phi_{\text {initial }}^{2} \sim O\left(\sqrt{\Delta E \cdot \gamma \cdot L_{P}} / \hbar^{2}\right)
\end{aligned}
$$

But Equation (21) and Equation (20) interplay and also give more substances to the use of Equation (19) with our guess of Equation (18) for the determination of the initial graviton frequency, which has to be at least of the order of $10^{\wedge} 45$ Hertz due to the fantastically small initial bubble of space time considered.

In doing so, we need to consider initial conditions so considered that Equation (20) and Equation (21) should be consistent with the inflaton and "gravity's breath" document by Corda [17]. In addition, Freeze's statement of initial con- 
ditions for inflaton, as given by [18] should be adhered to. It is also extremely important that the LIGO results, even if this is of relic gravitational waves, as seen by Abbott in [19] [20] [21], should not be contravened.

We conclude also with the hope that interpolating between the results of Equation (19), Equation (20) and Equation (21) will also in time confer answers as to the initial evaluative conditions for gravity as given in [22] by Corda. This also may in time with further analysis tie in with minimum values of entropy by further analysis of Equation (4) in further future analysis of this problem.

\section{Fund}

Work partially supported by National Nature Science Foundation of China grant No. 11375279.

\section{References}

[1] Downes, T.G. and Milburn, G.J. Optimal Quantum Estimation for Gravitation. arXiv: 1108.5220 [gr-qc].

[2] Unruh, W.G. (1986) Why Study Quantum Theory? Canadian Journal of Physics, 64, 128-130. https://doi.org/10.1139/p86-019

[3] Beckwith, A. (2016) Gedanken Experiment for Refining the Unruh Metric Tensor Uncertainty Principle via Schwarzschild Geometry and Planckian Space-Time with Initial Nonzero Entropy and Applying the Riemannian-Penrose Inequality and Initial Kinetic Energy for a Lower Bound to Graviton Mass (Massive Gravity). Journal of High Energy Physics, Gravitation and Cosmology, 2, 106-124. https://doi.org/10.4236/jhepgc.2016.21012

[4] Beckwith, A.W. Gedankenexperiment for Refining the Unruh Metric Tensor Uncertainty Principle via Schwartzshield Geometry and Planckian Space-Time with Initial Non Zero Entropy. Ukranian Journal of Physics (Unpublished). http://vixra.org/pdf/1509.0173v6.pdf

[5] Kolb, E. and Turner, M. (1990) The Early Universe. Addison-Wesley Publishing Company, Redwood City, California.

[6] Louis, J., Mohaupt, T. and Theisen, S. (2007) String Theory: An Overview. Lecture Notes in Physics, 721, 289-323.

http://www.aei.mpg.de/ theisen/LMT.pdf https://doi.org/10.1007/978-3-540-71117-9_12

[7] Ammon, M. and Ergmenger, J. (2015) Gauge/Gravity Duality, Foundations and Applications. Cambridge University Press, Cambridge. https://doi.org/10.1017/CBO9780511846373

[8] Becker, K., Becker, M. and Schwarz, J.H. (2007) String Theory and M-Theory: A Modern Introduction. Cambridge University Press, Cambridge.

[9] Giovannini, M. (2008) A Primer on the Physics of the Cosmic Microwave Background. World Press Scientific, Hackensack, New Jersey. https://doi.org/10.1142/6730

[10] Camara, C.S., de Garcia Maia, M.R., Carvalho, J.C. and Lima, J.A.S. (2004) Nonsingular FRW Cosmology and Non Linear Dynamics. Physical Review D, 69, 123504. https://doi.org/10.1103/PhysRevD.69.123504

[11] Rovelli, C. and Vidotto, F. (2015) Covariant Loop Quantum Gravity, an Elementary 
Introduction to Quantu Gravity and Spinfoam Theory. Cambridge University Press, Cambridge.

[12] Ashtekar, A., Berger, B., Isenberg, J. and Mac Callum, M. (2015) General Relativity and Gravitation, a Centennial Perspective. Cambridge University Press, Cambridge. https://doi.org/10.1017/CBO9781139583961

[13] Padmanabhan, T. (2005) Understanding Our Universe: Current Status, and Open Issues. World Scientific, P.T.E. Ltd., Singapore, 175-204. http://arxiv.org/abs/gr-qc/0503107

[14] https://ned.ipac.caltech.edu/level5/Sept02/Padmanabhan/Pad1_2.html

[15] https://ned.ipac.caltech.edu/level5/Sept02/Padmanabhan/Pad7.html

[16] Beckwith, A. (2017) Constraints, in Pre Planckian Space-Time via Padmabhan's Approximation Leading to Initial Inflaton Constraints. http://vixra.org/abs/1701.0333

[17] Corda, C. (2011) Primordial Gravity's Breath. Electronic Journal of Theoretical Physics, 9, 1-10. http://www.ejtp.com/articles/ejtpv9i26.pdf https://arxiv.org/abs/1110.1772

[18] Freese, K. (1992) Natural Inflaton. In: Nath, P. and Recucroft, S., Eds., Particles, Strings, and Cosmology, Northeastern University, World Scientific Publishing Company, Singapore, 408-428.

[19] Abbott, B.P., et al. (2016) Observation of Gravitational Waves from a Binary Black Hole Merger. Physical Review Letters, 116, Article ID: 061102.

[20] Abbott, B.P., et al. (2016) GW151226: Observation of Gravitational Waves from a 22-Solar-Mass Binary Black Hole Coalescence. Physical Review Letters, 116, Article ID: 241103.

[21] Abbott, B.P., et al. (2016) Tests of General Relativity with GW150914. https://arxiv.org/pdf/1602.03841.pdf

[22] Corda, C. (2009) Interferometric Detection of Gravitational Waves: The Definitive Test for General Relativity. International Journal of Modern Physics D, 18, 2275-2282. https://arxiv.org/abs/0905.2502

https://doi.org/10.1142/S0218271809015904 